\title{
Chini Equations and Isochronous Centers in Three-Dimensional Differential Systems
}

\author{
Marc Chamberland ${ }^{\dagger}$ and Armengol Gasull* \\ $\dagger$ Department of Mathematics and Statistics, Grinnell College, \\ Grinnell, IA, 50112, U.S.A., e-mail: chamberl@math.grinnell.edu \\ * Departament de Matemàtiques, Edifici Cc, Universitat Autònoma de Barcelona, \\ 08193 Bellaterra, Barcelona, Spain, e-mail: gasull@mat.uab.cat
}

\begin{abstract}
We study the number of limit cycles of $T$-periodic Chini equations and some generalized Abel equations and apply the results obtained to illustrate the existence of isochronous centers in three-dimensional autonomous differential systems.
\end{abstract}

Keywords: Abel equations, Chini equations, periodic orbits, limit cycles, isochronous centers.

2000 Mathematics Suject Classification: Primary: 34C25; Secondary: $37 C 10,37 C 27$.

\section{Introduction and main results}

Consider non-autonomous differential equations of the form

$$
\frac{d x}{d t}=a_{n}(t) x^{n}+a_{n-1}(t) x^{n-1}+\cdots+a_{1}(t) x+a_{0}(t),
$$

where $x \in \mathbb{R}, t \in[0, T]$ and $a_{0}, a_{1}, \ldots, a_{n}: \mathbb{R} \rightarrow \mathbb{R}$, are smooth $T$-periodic functions. The first part of this paper studies a subfamily of these equations and examines the number of solutions satisfying $x(0)=x(T)$. Note that equation (1) can be considered on the cylinder $\mathbb{R} \times[0, T]$ so the solutions satisfying $x(0)=x(T)$ are usually called periodic solutions. A periodic solution which is isolated from other periodic solutions of (1) is called a limit cycle of the differential equation.

*The second author is partially supported by a MCYT/FEDER grant number MTM2008-03437 and by a CIRIT grant number 2005SGR 00550. 
The problem of studying the number of limit cycles of (1) goes back to V.A. Pliss ([13]), N. G. Lloyd ([8]) and C. Pugh ([7]). Notice that equation (1) with $n=1$ (resp. $n=2$ ) is a linear equation (resp. a Riccati equation). It is well known that linear (resp. Riccati) equations have either a continuum of periodic solutions or at most 1 limit cycle (resp. 2 limit cycles); see, for example, [7, 9].

When $n=3$, equation (1) is called an Abel equation. It is known that when $a_{3}(t)$ does not change sign, the upper bound for the number of limit cycles of the Abel equation is three (see $[5,7,13]$ ) and moreover that this bound is sharp. On the other hand, when either $a_{3}(t)$ changes sign or $n \geq 4$ (even in the case $a_{n}(t) \equiv 1$ ) there is no general upper bound for the number of limit cycles of $(1)$; see $[4,6,7,12]$. The examples having many limit cycles are constructed by taking the functions $a_{j}(t)$ to be trigonometric polynomials and their degrees increase with the number of limit cycles.

This paper studies the number of limit cycles of $T$-periodic differential equations of the form

$$
\frac{d x}{d t}=a_{n}(t) x^{n}+a_{1}(t) x+a_{0}(t),
$$

which are usually called Chini equations, see [2]. Our main result is motivated by the note of Elias $([3])$ and the results of $[4,11]$ about the number of limit cycles of equation

$$
\frac{d x}{d t}=a_{n}(t) x^{n}+a_{2}(t) x^{2}+a_{1}(t) x+a_{0}(t) ;
$$

see Theorem 2. After a first version of this paper was written also appeared [1]. The result of Elias is introduced as a motivating example for students and asserts that the Abel differential equation

$$
\frac{d x}{d t}=-x^{3}+\sin (t)
$$

has exactly one globally attracting limit cycle. We prove:

Theorem 1. Consider the T-periodic Chini equation (2), where $a_{n}(t), a_{1}(t)$ and $a_{0}(t)$ are T-periodic $\mathcal{C}^{1}$ functions and $a_{n}(t)$ does not change sign. Then

(a) If $n \geq 3$ is odd, equation (2) has at most three limit cycles taking into account their multiplicities. Moreover this upper bound is sharp.

(b) If $n \geq 1$ is odd and $a_{1}(t) \equiv 0$, equation (2) has at most one limit cycle. If this limit cycle exists, it is hyperbolic and its stability is given by the sign of $a_{n}(t)$. Moreover, when $a_{n}(t)$ does not vanish the limit cycle always exists and it is a global attractor (resp. repeller) when the sign of $a_{n}(t)$ is negative (resp. positive).

(c) If $n \geq 2$ is even, equation (2) has at most two limit cycles taking into account their multiplicities. Moreover this upper bound is sharp. 
In Propositions 3 and 4 we prove that the hypothesis on $a_{n}(t)$ of the theorem can not be removed. Finally, in Section 3 we extend some of the above results to $T$-periodic equations of the form

$$
\frac{d x}{d t}=h(t)-f(x)
$$

and apply them to illustrate the existence of invariant surfaces formed by isochronous centers in some three dimensional autonomous systems; see Theorem 5.

\section{A family of Abel equations}

We recall the results of [4] to compare them with Theorem 1. Note that items (a) of both results coincide and it is stated in Theorem 1 only for the sake of completeness.

Theorem 2. ([4]) Consider the 1-periodic generalized Abel equation

$$
\frac{d x}{d t}=a_{n}(t) x^{n}+a_{2}(t) x^{2}+a_{1}(t) x+a_{0}(t)
$$

where $a_{n}(t), a_{2}(t), a_{1}(t)$ and $a_{0}(t)$ are $\mathcal{C}^{1}$ 1-periodic functions and $a_{n}(t)$ does not change sign. Then

(a) If $n \geq 3$ is odd, equation (4) has at most three limit cycles taking into account their multiplicities. Moreover this upper bound is sharp.

(b) If $n \geq 4$ is even, for any $\ell \in \mathbb{N}$, there is an equation of type (4) having at least $\ell$ limit cycles.

Proof of Theorem 1. (a) By using part (a) of Theorem 2, the result follows. Notice that to prove that the bound is sharp it suffices to consider the three functions $a_{i}(t) \equiv c_{i}$ for suitable constants $c_{i}, i=0,1, n$. Then the limit cycles are given by some constants solutions. The same approach works to produce the sharpness results in (b) and (c); we omit the details.

(b-c) Consider a smooth $T$-periodic ordinary differential equation

$$
\frac{d x}{d t}=S(t, x)
$$

defined in a neighborhood the strip $\{(t, x) \in[0, T] \times \mathbb{R}\}$ and the two straight lines $L_{0}:=\{(t, x): t=0\}$ and $L_{T}:=\{(t, x): t=T\}$. Whenever it is defined, we can consider the return map $h: L_{0} \rightarrow L_{T}$ given as follows: if $\rho \in L_{0}$, then $h(\rho)=\varphi(T ; \rho)$, where $x=\varphi(T ; \rho)$ denotes the solution of $(5)$ such that $\varphi(0 ; \rho)=\rho$. With this definition the limit cycles of the differential equation correspond with the initial conditions that the isolated solutions of the function $h(\rho)-\rho$. Moreover the 
multiplicity of any limit cycle is the multiplicity of $\rho$ as a zero of $h(\rho)-\rho$. Simple solutions are called hyperbolic limit cycles. It is not difficult to prove that

$$
\begin{aligned}
h^{\prime}(\rho) & =\exp \int_{0}^{T} \frac{\partial S}{\partial x}(t, \varphi(t ; \rho)) d t \\
h^{\prime \prime}(\rho) & =h^{\prime}(\rho)\left[\int_{0}^{T} \frac{\partial^{2} S}{\partial x^{2}}(t, \varphi(t ; \rho)) \exp \left\{\int_{0}^{t} \frac{\partial S}{\partial x}(s, \varphi(s ; \rho)) d s\right\} d t\right]
\end{aligned}
$$

see, for example, [10]. By applying the above formulas to equation (2) we obtain

$$
\begin{aligned}
h^{\prime}(\rho) & =\exp \int_{0}^{T}\left(n a_{n}(t)(\varphi(t ; \rho))^{n-1}+a_{1}(t)\right) d t \\
h^{\prime \prime}(\rho) & =h^{\prime}(\rho)\left[\int_{0}^{T} n(n-1) a_{n}(t)(\varphi(t ; \rho))^{n-2} \times\right. \\
& \left.\exp \left\{\int_{0}^{t}\left(n a_{n}(s)(\varphi(s ; \rho))^{n-1}+a_{1}(s)\right) d s\right\} d t\right] .
\end{aligned}
$$

When $n$ is odd and $a_{1}(t) \equiv 0$ it is clear that $h^{\prime}(\rho)<1$ or $h^{\prime}(\rho)>1$ according whether $a_{n}(t) \leq 0$ or $a_{n}(t) \geq 0$. Therefore $h(\rho)=\rho$ has at most one zero, and when it exists it is hyperbolic, as desired. When $a_{n}(t)$ does not vanish, the existence of the limit cycle follows by considering the vector field associated to the differential equation on the lines $\{x= \pm M\}$ for sufficiently large $M$.

When $n$ is even it is clear that $h^{\prime \prime}(\rho)$ does not vanish and the existence of at most two solutions of the equation $h(\rho)=\rho$ (taking the multiplicities into account) follows from Rolle's Theorem.

Simple examples having non-constant limit cycles and reaching the upper bound predicted by Theorem 1 can also be easily constructed. For instance, the differential equation

$$
x^{\prime}=\sin (t)-x\left(x^{2}-3\right)
$$

has exactly three limit cycles. Theorem 1 shows that this differential equation has at most three limit cycles, so the goal is to show it has at least three. To do so, note that $x^{\prime} \geq 1$ when $x=1$ and $x^{\prime} \leq-17$ when $x=3$. Continuity of solutions implies that there is a periodic solution whose initial value starts between 1 and 3 (consider the value of the solution at $t=2 \pi$ ). A continuum of solutions cannot exist since the solution starting at 1 or 3 cannot be periodic. Thus, this periodic solution is a limit cycle. Similarly, one may produce a limit cycle between $x=-1$ and $x=1$ as well as $x=-3$ and $x=-1$.

We also want to remark that the proof of part (b) of Theorem 1 can also be done by imitating the proof in [3]. First we prove the inequality

$$
u^{n}-v^{n} \geq(u-v)^{n} / 2^{n-1}, \quad \text { when } u \geq v \text { and } n \text { odd. }
$$

This can be done by showing that the absolut minimum in $\mathbb{R}$ of the function $\left(x^{n}-\right.$ $1) /(x-1)^{n}$ when $n$ is odd is at $x=-1$ and is $1 / 2^{n-1}$. Note that the above 
inequality does not hold when $n$ is even. If $u(t)>v(t)$ are two periodic solutions of $d x / d t=a_{n}(t) x^{n}+a_{0}(t)$ and assuming without loss of generality that $a_{n}(t) \leq 0$, we have

$$
(u(t)-v(t))^{\prime}=a_{n}(t)\left(u(t)^{n}-v(t)^{n}\right) \leq a_{n}(t)(u(t)-v(t))^{n} / 2^{n-1}
$$

for all $t \in \mathbb{R}$. Integrating the inequality

$$
\frac{(u(t)-v(t))^{\prime}}{(u(t)-v(t))^{n}} \leq \frac{a_{n}(t)}{2^{n-1}}
$$

from 0 to $T$ yields a contradiction. Our proof has the advantage that it treats both cases ( $n$ even and odd) together and gives the hyperbolicity of the limit cycle.

The next results show that the hypothesis on $a_{n}(t)$ in Theorem 1 is essential.

Proposition 3. Given any $\ell \in \mathbb{N}$, and for each $n \geq 3$, there are Chini equations of the form (2),

$$
\frac{d x}{d t}=a_{n}(t) x^{n}+a_{0}(t)
$$

with $a_{n}(t)$ and $a_{0}(t)$ smooth T-periodic functions and $a_{n}(t)$ changing sign, having at least $\ell$ limit cycles.

Proof. Without loss of generality we can take $T=1$. Consider the differential equation

$$
\frac{d x}{d t}=2 \pi \cos (2 \pi t) x^{n}+\varepsilon b(t),
$$

where $b(t)$ is a 1-periodic trigonometrical polynomial to be determined, and $\varepsilon$ is a small parameter. Write the solution $x=\varphi(t ; \rho, \varepsilon)$ such that $\varphi(0 ; \rho, \varepsilon)=\rho$ as

$$
x=\varphi(t ; \rho, \varepsilon)=u(t, \rho)+v(t, \rho) \varepsilon+O\left(\varepsilon^{2}\right) .
$$

Plugging this expression into (6) yields

$$
\begin{aligned}
& u(t, \rho)=\rho\left(1+(1-n) \sin (2 \pi t) \rho^{n-1}\right)^{1 /(1-n)}, \\
& v(t, \rho)=\left(\frac{u(t, \rho)}{\rho}\right)^{n} \int_{0}^{t} b(t)\left(1+(1-n) \sin (2 \pi t) \rho^{n-1}\right)^{n /(n-1)} d t .
\end{aligned}
$$

For $t=1$ we get

$$
\varphi(1 ; \rho, \varepsilon)=\rho+V(\rho) \varepsilon+O\left(\varepsilon^{2}\right),
$$

where

$$
V(\rho):=\int_{0}^{1} b(t)\left(1+(1-n) \sin (2 \pi t) \rho^{n-1}\right)^{n /(n-1)} d t .
$$

At this point we can follow the technique developed in [7], (see also [4]) and prove that given any arbitrary polynomial of degree $k, p_{k}(y)$, there exists $b(t)$ of the form

$$
b(t)=\sum_{j=0}^{k} \beta_{j} \sin ^{j}(2 \pi t) \quad \text { with } \quad \beta_{j} \in \mathbb{R},
$$


such that

$$
V(\rho)=p_{k}\left(\rho^{n-1}\right)+O\left(\rho^{k(n-1)+1}\right) .
$$

By using again the results of [7], an appropriate choice of $k$ and $p_{k}$ produces the equation

$$
\frac{\varphi(1 ; \rho, \varepsilon)-\rho}{\varepsilon}=p_{k}\left(\rho^{n-1}\right)+O\left(\rho^{k(n-1)+1}\right)+O(\varepsilon)=0
$$

which has at least $\ell$ real isolated zeros in a neighborhood of the origin, and so the Chini equation has at least $\ell$ limit cycles, as we wanted to prove.

Proposition 4. Let $g(t)$ be a non-constant smooth $T$-periodic function, $m \in \mathbb{N}$ and $k \in \mathbb{R}$ such that $g^{2 m}(t)-k^{2 m}$ does not vanish. Then the differential equation

$$
\frac{d x}{d t}=\frac{g^{\prime}(t)}{g^{2 m}(t)-k^{2 m}}\left(x^{2 m}-k^{2 m}\right)
$$

has a continuum of periodic solutions (including the three explicit solutions $x=k$, $x=-k$ and $x=g(t))$ and $a_{2 m}(t)=g^{\prime}(t) /\left(g^{2 m}(t)-k^{2 m}\right)$ changes sign in $[0, T]$.

Proof. The three explicit solutions are obvious. Define the function $H(x)$ implicitly by $H^{\prime}(x)=1 /\left(x^{2 m}-k^{2 m}\right)$. If $x=x(t)$ is a solution to (7), then one may easily show that $H(g(t))-H(x(t))=C$, for some constant $C$, and hence one has a continuum of solutions. The function $a_{2 m}(t)$ changes sign as a consequence of the condition $g(0)=g(T)$.

Other examples having three given non-constant periodic orbits are not difficult to construct. For instance we can obtain an explicit Abel equation of the form (2) with $n=4$ by imposing that the periodic functions $x=k+\sin (t), x=-k-\sin (t)$ and $x=\sin (t)$, for $k>1$, are three of its solutions.

\section{Isochronous Centers in Three-Dimensional Differential Systems}

In this section we will construct a differential system in $\mathbb{R}^{3}$ from a generalized Abel equation which exhibits a continuum of isochronous periodic orbits. This requires a generalization of Theorem 1 to the equation

$$
\frac{d x}{d t}=A g(t)-f(x) .
$$

Theorem 5. Let $f$ be a $\mathcal{C}^{1}$ function satisfying $f(0)=0, g(t)$ be a $T$-periodic function and $A \in \mathbb{R}$. Then

(a) Suppose $f^{\prime}(x)>0$ (resp. <0) for sufficiently small non-zero $x$. Then for all sufficiently small $A$, equation (8) has a locally attracting (resp. repelling) T-periodic solution. 
(b) Suppose that $f^{\prime}(x)>0$ (resp. $<0$ ) for all $x \in \mathbb{R} \backslash\{0\}$, then the equation (8) has at most one periodic orbit and when it exists it is a T-periodic hyperbolic stable (resp. unstable) limit cycle. Moreover if $\lim _{x \rightarrow \pm \infty}|f(x)|=\infty$ the limit cycle always exists and is a global attractor (resp. repeller).

Proof. (a) If $f^{\prime}(x)>0$ for sufficiently small non-zero $x$, for sufficiently small $A$, there exists $x_{1}>0$ such that $\varphi\left(T ; x_{1}\right)<x_{1}$ and $\varphi\left(T ;-x_{1}\right)>-x_{1}$. This implies the existence of a limit cycle and that $|\varphi(t ; \rho)|<x_{1}$ for all $t>0$ and $|\rho|<x_{1}$. As in the proof of Theorem $1, h^{\prime}(\rho)<1$ and hence the limit cycle is attractive. If $f^{\prime}(x)<0$ for sufficiently small non-zero $x$, the proof is the same except that the limit cycle is repelling.

(b) Assume for instance that $f^{\prime}(x)>0$ for all non-zero $x$. By using again that

$$
h^{\prime}(\rho)=\exp \int_{0}^{T} \frac{\partial S}{\partial x}(t, \varphi(t ; \rho)) d t=\exp \left(-\int_{0}^{T} f^{\prime}(\varphi(t ; \rho)) d t\right)<1
$$

we get the uniqueness and stability of the periodic orbit. To prove the existence it suffices to study the flow of the lines $x= \pm M$ for $M$ sufficiently large.

Let us consider the particular case of equation (8)

$$
\frac{d x}{d t}=A \sin (t+c)-f(x), \quad f(0)=0 .
$$

By letting $y=A \sin (t+c)$ and $z=A \cos (t+c)$, equation (9) may be written as the three-dimensional system

$$
\left\{\begin{array}{l}
x^{\prime}=y-f(x), \\
y^{\prime}=z, \\
z^{\prime}=-y
\end{array}\right.
$$

for any $A$ and $c$. Theorem 5 , with its conditions on $f$, guarantees the existence of limit cycles in (9) for each $A$ and $c$. Changing $c$ (equivalent to shifting $t$ ) while keeping $A$ fixed will yield the same limit cycle. Letting $c$ range from 0 to $2 \pi$ while keeping $A$ fixed generates the $2 \pi$-periodic limit cycle in three-dimensional phase space. Note that $A=0$ corresponds to the case with an equilibrium point at the origin. The function $H(x, y, z)=y^{2}+z^{2}$ is a first integral and one of its level sets corresponds to the stable (resp. unstable) manifold of the limit cycle when $f^{\prime}(x)>0$ (resp. $f^{\prime}(x)<0$ ) for small non-zero $x$.

Combining these limit cycles for each appropriate $A$ generates a two-dimensional manifold which is an isochronous center. Note that in the case $f^{\prime}(0)=0$ all the eigenvalues of the Jacobian at the origin have zero real part, hence the linear theory cannot be used to predict invariant manifolds. Finding an invariant corresponding 
to this surface is not as simple as the function $H$, but may be found by writing $x$ as a power series in $y$ and $z$, namely

$$
x=a_{1,0} y+a_{0,1} z+a_{2,0} y^{2}+a_{1,1} y z+a_{0,2} z^{2}+O\left(r^{3}\right),
$$

where $r=\sqrt{y^{2}+z^{2}}$. Placing this into the equation $x^{\prime}=y-f(x)$ and matching coefficients yields the Taylor expansion for the isochronous center. For example, we compute the coefficients for the problem of Elias which corresponds to equation (9) with $A=1, c=0$ and $f(x)=x^{3}$. Imposing the relation up to the degree 3 terms yields

$$
x=-z+a_{2,0}\left(y^{2}+z^{2}\right)+\frac{2}{3} y^{3}+y z^{2}+O\left(r^{4}\right)
$$

where the constant $a_{2,0}$ has to be determined by exploring higher powers of $y$ and $z$. Doing two more steps gives $a_{2,0}=0$ and

$$
x=-z+\frac{2}{3} y^{3}+y z^{2}+a_{4,0}\left(y^{2}+z^{2}\right)^{2}+\frac{2}{3} y^{2} z^{3}+\frac{13}{15} z^{5}+O\left(r^{6}\right) .
$$

Similarly, $a_{4,0}=0$ and

$$
\begin{aligned}
x= & -z+\frac{2}{3} y^{3}+y z^{2}+\frac{2}{3} y^{2} z^{3}+\frac{13}{15} z^{5}+a_{6,0}\left(z^{2}+y^{2}\right)^{3} \\
& -\frac{52}{75} y^{7}-\frac{232}{75} y^{5} z^{2}-\frac{73}{15} y^{3} z^{4}-\frac{13}{5} y z^{6}+O\left(r^{8}\right),
\end{aligned}
$$

where $a_{6,0}$ can be subsequently determined. Observe that if the function $f$ is nonlinear, then the expression of $x$ is complicated and seems not be polynomial.

Notice also that when $f(x)=x^{2 m}$, by using Theorem 1.(c) there can exist cylinders without periodic orbits and other cylinders with two limit cycles. This scenario produces a more complicated geometry of the isochronous surfaces.

There is also a different and natural way of obtaining a three vector field that contains the dynamics of the generalized Abel equation (9). Taking two derivatives in the equation and adding gives

$$
x^{\prime \prime \prime}+x^{\prime}=-f(x)-f^{\prime \prime}(x)\left(x^{\prime}\right)^{2}-f^{\prime}(x) x^{\prime \prime} .
$$

Letting $v=x^{\prime}$ and $w=v^{\prime}=x^{\prime \prime}$ produces the system

$$
\left\{\begin{array}{l}
x^{\prime}=v \\
v^{\prime}=w \\
w^{\prime}=-v-f(x)-f^{\prime \prime}(x) v^{2}-f^{\prime}(x) w
\end{array}\right.
$$

It is easy to check that performing the change of variables

$$
x=x, \quad y=v+f(x), \quad z=w+f^{\prime}(x) v
$$

transforms (11) into (10). 


\subsection{Isochronous centers in higher dimensions}

It is not difficult to extend the previous results to higher dimensions following the second approach. We introduce the following operator $D:=\frac{d}{d t}$ and for any polynomial of degree $m$ with real constant coefficients $p(x)=c_{m} x^{m}+c_{m-1} x^{m-1}+\cdots+c_{1} x+c_{0}$, then

$p(D)=c_{m} D^{m}+c_{m-1} D^{m-1}+\cdots+c_{1} D+c_{0} I d=c_{m} \frac{d^{m}}{d t^{m}}+c_{m-1} \frac{d^{m-1}}{d t^{m-1}}+\cdots+c_{1} \frac{d}{d t}+c_{0} I d$.

Consider an equation of the form

$$
\frac{d x}{d t}=h(t)-f(x)
$$

where $h(t)$ is such that $p(D)[h]=0$ for some polynomial $p$. Note that for equation (9) we have taken $p(x)=x^{2}+1$. For all this type of equations we get that

$$
p(D)\left[x^{\prime}\right]=p(D)[h(t)-f(x)]=-p(D)[f(x)]
$$

which gives rise to an ordinary differential equation of order $m+1$ and consequently to a system of equations in $\mathbb{R}^{m+1}$. Notice that if we want that $h(t)$ is $2 \pi$-periodic an easy way is to take as $h(t)$ a truncated Fourier series and then

$$
p(x)=\left(x^{2}+1\right)\left(x^{2}+2^{2}\right) \cdots\left(x^{2}+(k-1)^{2}\right)\left(x^{2}+k^{2}\right),
$$

for some $k \in \mathbb{N}$. For instance, if we consider equation

$$
x^{\prime}=A \sin (t)+B \sin (2 t)-f(x),
$$

we can apply the operator $p(D)=\left(D^{2}+I d\right)\left(D^{2}+4 I d\right)$ to the above expression giving rise to a differential system in $\mathbb{R}^{5}$.

\section{References}

[1] A. Álvarez, J. L. Bravo And M. Fernández. The number of limit cycles for generalized Abel equations with periodic coefficients of definite sign, Commun. Pure Appl. Anal. 8, 1493-1501 (2009).

[2] M. Chini. Sull'integrazione di alcune equazioni differenziali del primo ordine, Rediconti Instituto Lombardo (2) 57, 506-511 (1924).

[3] U. Elias. Qualitative Analysis of a Differential Equation of Abel, Amer. Math. Monthly 115, 147-149 (2008).

[4] A. Gasull And A. Guillamon. Limit cycles for generalized Abel equations, Internat. J. Bifur. Chaos Appl. Sci. Engrg. 16, 3737-3745 (2006). 
[5] A. Gasull and J. Llibre. Limit cycles for a class of Abel equations, SIAM J. Math. Anal. 21, 1235-1244 (1990).

[6] Y. IL'YASHENKo. Hilbert-type numbers for Abel equations, growth and zeros of holomorphic functions, Nonlinearity 13, 1337-1342 (2000).

[7] A. Lins Neto. On the number of solutions of the equation $d x / d t=$ $\sum_{j=0}^{n} a_{j}(t) x^{j}, 0 \leq t \leq 1$ for which $x(0)=x(1)$, Inv. Math. 59, 67-76 (1980).

[8] N. G. Lloyd. The number of periodic solutions of the equation $\dot{z}=z^{N}+$ $p_{1}(t) z^{N-1}+\cdots+p_{N}(t)$, Proc. London Math. Soc. 27, 667-700 (1973).

[9] N. G. Lloyd. On a class of differential equations of Riccati type, J. London Math. Soc. 10, 1-10 (1975).

[10] N. G. Lloyd. A note on the number of limit cycles in certain two-dimensional systems, J. London Math. Soc. 20, 277-286 (1979).

[11] A. A. PAnov. The number of periodic solutions of polynomial differential equations, Math. Notes 64, 622-628 (1998).

[12] A. A. Panov. On the diversity of Poincaré mappings for cubic equations with variable coefficients, Funct. Anal. and its Appl. 33, 310-312 (1999).

[13] V.A. PLiss. Non local problems of the theory of oscillations, Academic Press, New York, 1966. 